\title{
Antibiotic-Resistant Spondylodiscitis With Canal Invasion and Aggressive Evolution to Epidural Abscess: A Case Series of Spontaneous Occurrence in 16 Patients
}

\author{
PHILIP ROSINSKY, MD, ${ }^{1}$ SHAY MANDLER, MD,${ }^{1}$ NIV NETZER, MD,${ }^{1}$ MEITAL ADY, MD,${ }^{2}$ DANIELLE \\ ELMALIACHE, MA, ${ }^{1}$ SHAUL SAGIV, MD ${ }^{1}$ PELEG BEN-GALIM, MD ${ }^{1}$ \\ Departments of ${ }^{1}$ Orthopaedic Surgery and ${ }^{2}$ Imaging, Kaplan Medical Center, Rehovot, Israel
}

\begin{abstract}
Background: The study aimed to assess the effectiveness of antibiotic treatment for spondylodiscitis, its failure rates, and the need for surgical intervention.

Methods: This is a retrospective study of patients who presented with spontaneous deep spinal infections and spondylodiscitis between 2011 and 2013. Clinical, bacteriologic, and radiographic data during hospitalization were analyzed.

Results: A total of 16 patients presented with deep spinal infections during the study period; 15 of them presented with spontaneous pyogenic spondylodiscitis, and 1 presented with epidural abscess. Median age was 68 years (range, 5080 years), and $6(38 \%)$ were healthy young laborers. None of the patients were immunocompromised. On admission all patients presented with pain, there was fever in 3 patients $(19 \%)$, and there was elevated blood C-reactive protein, white blood cell count and erythrocyte sedimentation rate, with a mean of $147 \pm 83.1 \mathrm{mg} / \mathrm{L}, 11.65 \pm 5.6 \times 10^{3} / \mu \mathrm{L}, 93.6 \pm$ $35.1 \mathrm{~mm} / \mathrm{h}$, respectively. A total of 15 patients $(94 \%)$ developed infections that were refractory to appropriate culturespecific intravenous antibiotic treatment (mean, 10.2 days); 8 patients $(50 \%)$ deteriorated neurologically and required wide surgical decompression. Complications included widespread epidural free gas in 2 patients $(12 \%)$, multiple bilateral psoas abscesses in 2 patients (12\%), kyphotic segmental instability in 4 patients $(25 \%)$, and inferior vena cava septic thrombi in 1 patient (6\%). A total of 3 patients (19\%) died within 6 months; 7 of 13 surviving patients still had residual neurologic deficits at the 6-month follow-up.

Conclusions: Spondylodiscitis may be resistant to antibiotic treatment and may evolve into epidural abscess via extension of the infection and pus into the spinal canal, necessitating repetitive surgical treatment due to neurologic and clinical deterioration, and expansion of the persistent infection with a mass effect. Increased vigilance for this condition and its misleading initial presentations is warranted.
\end{abstract}

Other \& Special Categories

Keywords: antibiotic resistance, spondylodiscitis, spinal epidural abscess

\section{INTRODUCTION}

Spondylodiscitis, also referred to as pyogenic discitis and vertebral osteomyelitis, is defined as an infection limited to the intervertebral disc (discitis) and adjacent vertebrae (vertebral osteomyelitis). The incidence of spondylodiscitis has been estimated at 2.4 cases per 100000 people in the population. ${ }^{1}$

The disease course is often benign, and in the vast majority of cases it resolves with appropriate antibiotic treatment alone. ${ }^{2-4}$ The more aggressive spinal epidural abscess (SEA) is usually characterized by the accumulation of purulent material posterior to the dura, that is, between the dura and the osseoligamentous confines of the posterior spinal canal, often requiring surgical decompression and drainage and antibiotic treatment. ${ }^{5} \mathrm{~A}$ number of bacteriologic agents, including fungi, have been isolated, with Staphylococcus aureus being the most common, responsible for approximately $50 \%$ of cases. $^{6}$

Typically, patients with deep spinal infection will have predisposing factors, including elderly age, malnutrition, immunocompromised status (such as intravenous drug abuse, HIV, malignancy, chronic steroid usage, diabetes mellitus, etc), septicemia, recent spinal surgery, and intravascular devices. ${ }^{2}$ Resolution of the disc and bone infection with antibiotic treatment for spondylodiscitis is expected. On the other hand, if the infectious process 
continues to progress despite antibiotic treatment, waiting until manifestation of the "classic triad"fever, back pain, and neurologic deficit - can be catastrophic, because at this point the damage to the spinal cord or nerve roots may be irreversible. ${ }^{7}$

The purpose of this study is to assess the effectiveness of antibiotic treatment for spondylodiscitis, its failure rates, and the need for surgical intervention.

\section{MATERIALS AND METHODS}

A retrospective review of prospectively collected data was performed on all cases of spondylodiscitis or SEA treated at our institution between 2011 and 2013. Institutional Review Board approval was obtained for this study. Inclusion criteria included acute bacterial spontaneous spondylodiscitis or SEA in an adult patient (older than 18 years) who presented with spontaneous acute bacterial spondylodiscitis or pyogenic SEA without predisposing spinal injections or surgical procedures in the prior 2 years. Exclusion criteria included children; any prior recent spinal surgery, injection, or invasive intervention within the past 2 years; intravenous (IV) treatments or blood infusions within the past year.

A total of 16 patients were identified by a query of our institutional database using the following diagnostic codes: SEA, spinal cord infection, vertebral osteomyelitis, discitis, and back abscess. All cases were confirmed by increased inflammatory markers, radiologic studies, and blood or tissue cultures. A supplementary search using the nonspecific lesion codes for acute paraplegia, acute tetraplegia, and cauda equina syndrome was also completed. Failure of antibiotic treatment was defined as appearance of neurologic deterioration, spinal instability, or continuous clinical and/or radiographic deterioration despite inward IV antibiotic treatment necessitating surgical treatment. Radiographic deterioration was not considered as a stand-alone indication of antibiotic failure but was considered when in combination with clinical or neurologic deterioration and included: (1) newly developing instability and (2) infectious process and pus evidently seen entering the canal and epidural space on subsequent magnetic resonance imaging (MRI).

Patients' data were gathered and reviewed via inhospital electronic and chart clinical notes, imaging studies, and electronic records. Data included: demographics, medical history, emergency room admission, hospitalization charts, hospital discharge summaries, clinic follow-up notes, and radiology. Laboratory results were reviewed and inflammatory markers, including C-reactive protein, white blood cell count, and erythrocyte sedimentation rate were recorded. Bacteriology records were reviewed for positive blood cultures and/or intraoperative tissue cultures, the infectious agent, and relevant culturespecific antibiotic treatment, dosage, and duration were recorded. Length of hospitalization and the number of surgeries patients underwent were also noted. Radiologic investigations were reviewed, the infection was characterized by its location (cervical, thoracic, lumbar, or panspinal), and complications such as psoas abscesses, inferior vena cava septic thrombi, and spinal column instability were noted. Neurologic status upon discharge and at the clinic follow-up visits was noted.

\section{RESULTS}

A total of 16 consecutive adult patients met the criteria and were identified with spontaneous acute bacterial spondylodiscitis or pyogenic SEA. A total of 15 patients $(94 \%)$ initially had spondylodiscitis anterior to the posterior longitudinal ligament (PLL) and 1 patient had SEA appearing in the dorsal canal space posterior to the dura, with extension of the SEA into the subdural space. The mean age at presentation was 68 years (range, 50-80 years), and 9 patients $(56 \%)$ were male. None of the patients were immunocompromised, and 6 of the 16 patients $(38 \%)$ were healthy young laborers who had no prior comorbidities (Table). Initial presentation included fever in 3 patients $(18.75 \%)$. Inflammatory markers were elevated as reviewed in blood C-reactive protein concentration, white blood cell count, and erythrocyte sedimentation rate laboratory results, with mean values of $147 \pm 83.1$ $\mathrm{mg} / \mathrm{L}, 11.65 \pm 5.6 \times 10^{3} / \mu \mathrm{L}$, and $93.6 \pm 35.1 \mathrm{~mm} / \mathrm{h}$, respectively (Table). In 9 of 16 patients $(56 \%)$ the infection was localized in the lumbar spine, in 5 of $16(31 \%)$ the infection was at the thoracolumbar junction, and 2 of $16(13 \%)$ had a multilevel disease. All patients were initially treated with IV antibiotics following multiple blood- and/or CT-guided local tissue cultures.

Failure of IV antibiotic treatment was evident in 15 of 16 patients $(94 \%)$, who developed infections that were refractory to appropriate culture-specific IV antibiotic treatment, deteriorated neurologically or biomechanically, and required wide surgical 


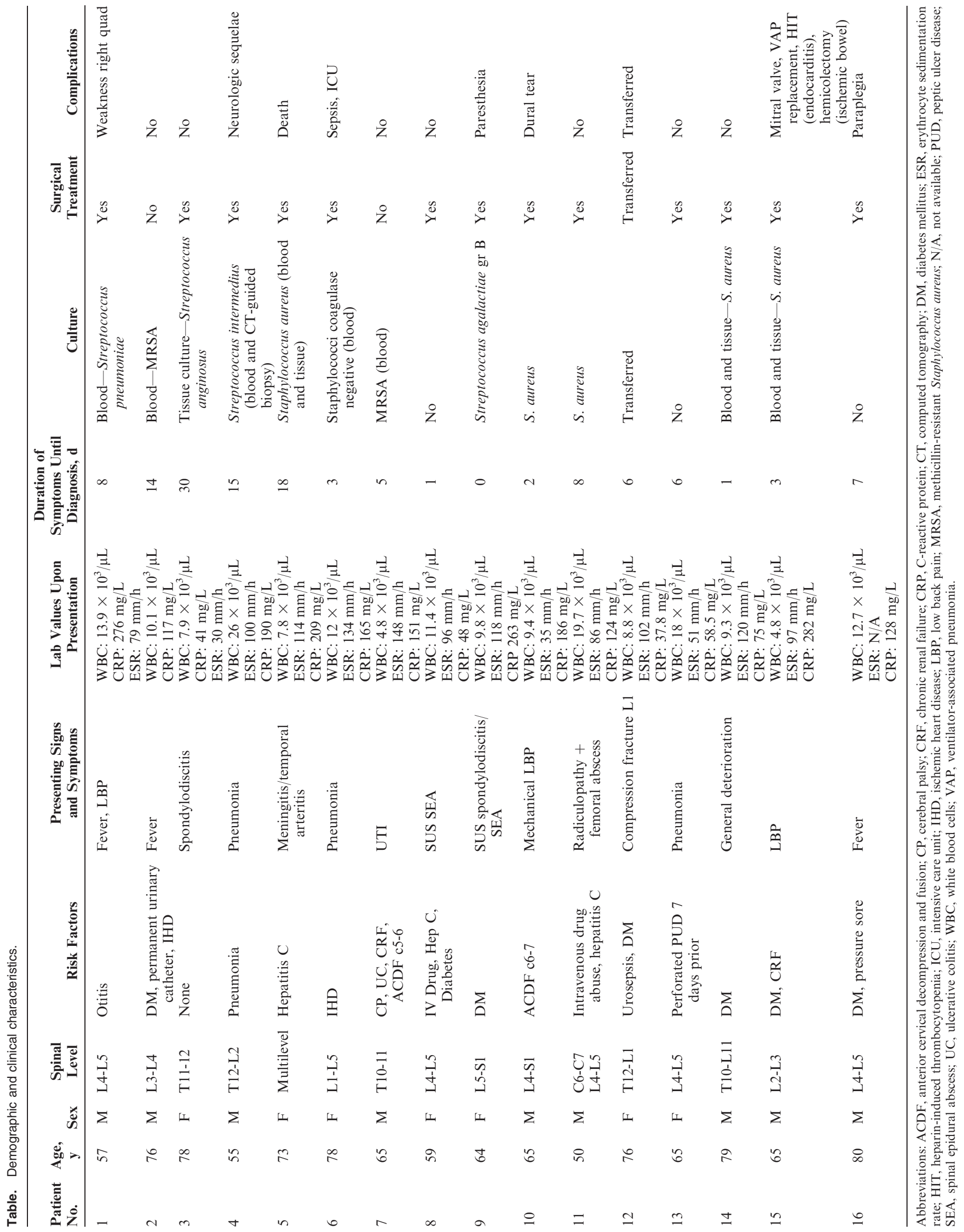




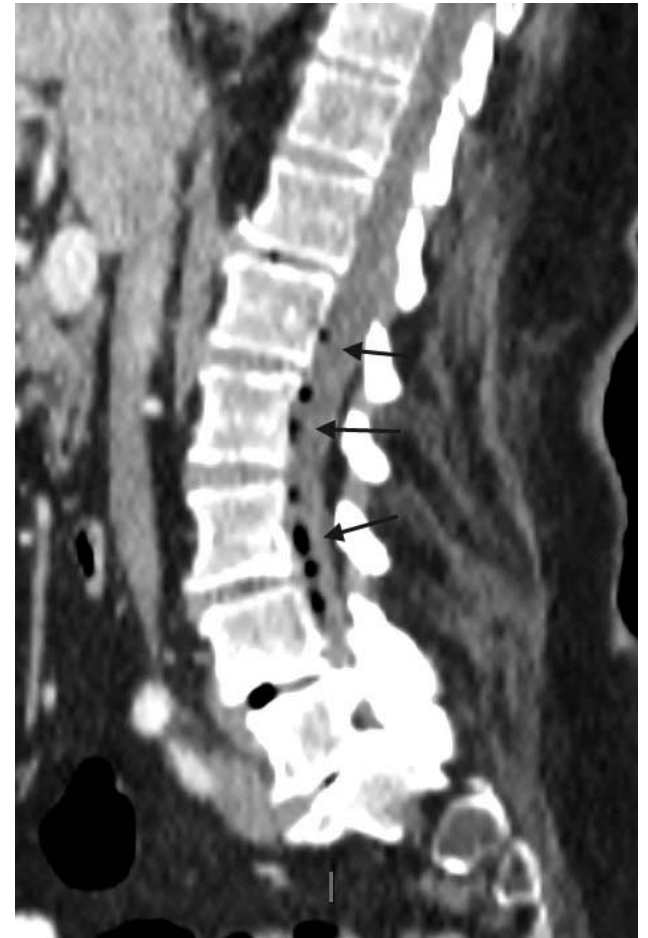

Figure 1. Epidural free gas. A 77-year-old woman who was previously healthy presented with fever, vomiting, and weakness and received a diagnosis of pneumonia. While under intravenous antibiotics she developed lower back pain and bilateral leg weakness. A subtle hypodense stripe in the spinal canal was present, suggesting an epidural abscess that was confirmed on magnetic resonance imaging. Note the free gas air bubbles (gray arrows) on computed tomography. The patient underwent surgical drainage of the epidural abscess.

decompression, debridement, and stabilization, followed by long-term antibiotics and rehabilitation. The average course of IV antibiotics prior to deterioration necessitating surgical intervention was 10.2 days. Overall hospitalizations averaged 41.6 days, and 3 patients $(18.75 \%)$ required intensive care unit (ICU) treatment. Radiographic deterioration with extension of the intradiscal infectious phlegmon through the posterior disc annulus, into the spinal canal, was apparent on subsequent MRI scans in all but 1 of these patients (Table). This radiographic deterioration depicts the fulminant nature of this spondylodiscitis, with complications including widespread epidural free gas in 2 patients (12\%; Figure 1), multiple bilateral psoas abscesses in 2 patients (12.5\%; Figure 2), kyphotic segmental instability in 4 patients $(25 \%$; Figure 3$)$, and inferior vena cava septic thrombi in 1 patient (6\%; Figure 4a).

Neurologic deterioration and compromise occurred in 8 of these 16 patients $(50 \%)$, including acute infectious qauda equina syndrome and paraplegia, during hospitalization and despite antibiotic

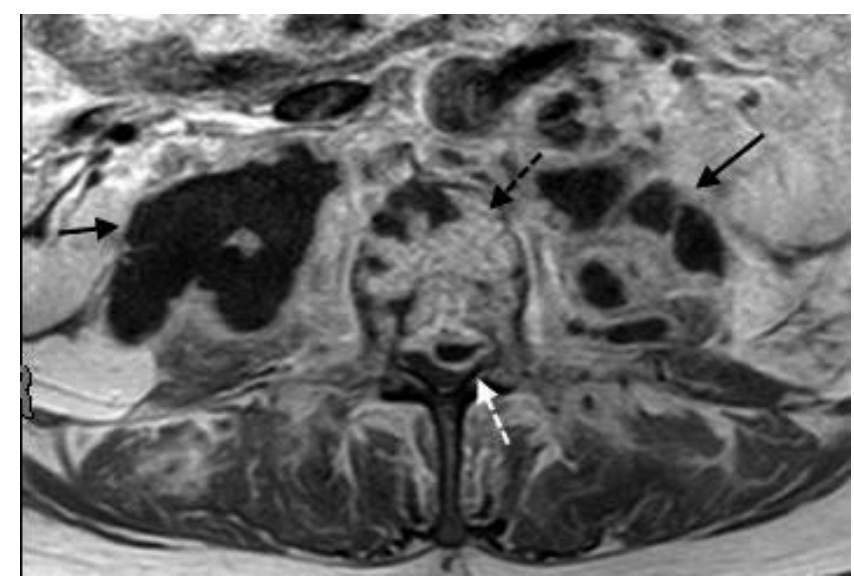

Figure 2. Bilateral psoas abscesses. This patient initially presented with fever and back pain with discitis on computed tomography scan. Culture-specific intravenous antibiotics were initiated, with an initial improvement of systemic signs, only to be followed by sudden appearance of paraparesis and bladder incontinence. Magnetic resonance imaging at this stage revealed extensive vertebral osteomyelitis and discitis (black dashed arrow) with posterior extension of the infection through the posterior annulus into the epidural space (white dashed arrow). Note also the multiple bilateral psoas abscesses (black arrows).

treatment. Some residual neurologic deficits were still present in 7 of 13 surviving patients $(44 \%)$ at the 6-month follow-up. Surgical decompression was performed in 15 patients $(94 \%)$, and 3 patients $(18.75 \%)$ required repetitive surgical debridement because of continued clinical deterioration. A total of 3 patients $(18.75 \%)$ died within 6 months. Blood cultures were positive in 11 patients $(69 \%)$, and in 1 patient $(6 \%)$ only tissue samples obtained during surgery were positive. A total of 4 patients $(25 \%)$ did not have positive blood or tissue cultures, although 1 of these patients was transferred to another hospital prior to surgical treatment and biopsy. The most common pathogen was $S$. aureus in 7 patients $(44 \%)$, with 2 of these being MRSA (Table; Figure 5).

\section{DISCUSSION}

Spondylodiscitis is a well-known entity that is typically considered a benign disease treated nonsurgically in the medicine wards in elderly and immunocompromised patients. Treatment is conservative, with long-term IV antibiotics, bed rest, and, in selected cases, casts and orthotics. This study reports on a consecutive series of patients presenting with a complicated clinical course of spondylodiscitis that begins as a seemingly benign disease involving the disc and adjacent vertebrae end plates; the disease was expected to improve and eventually 

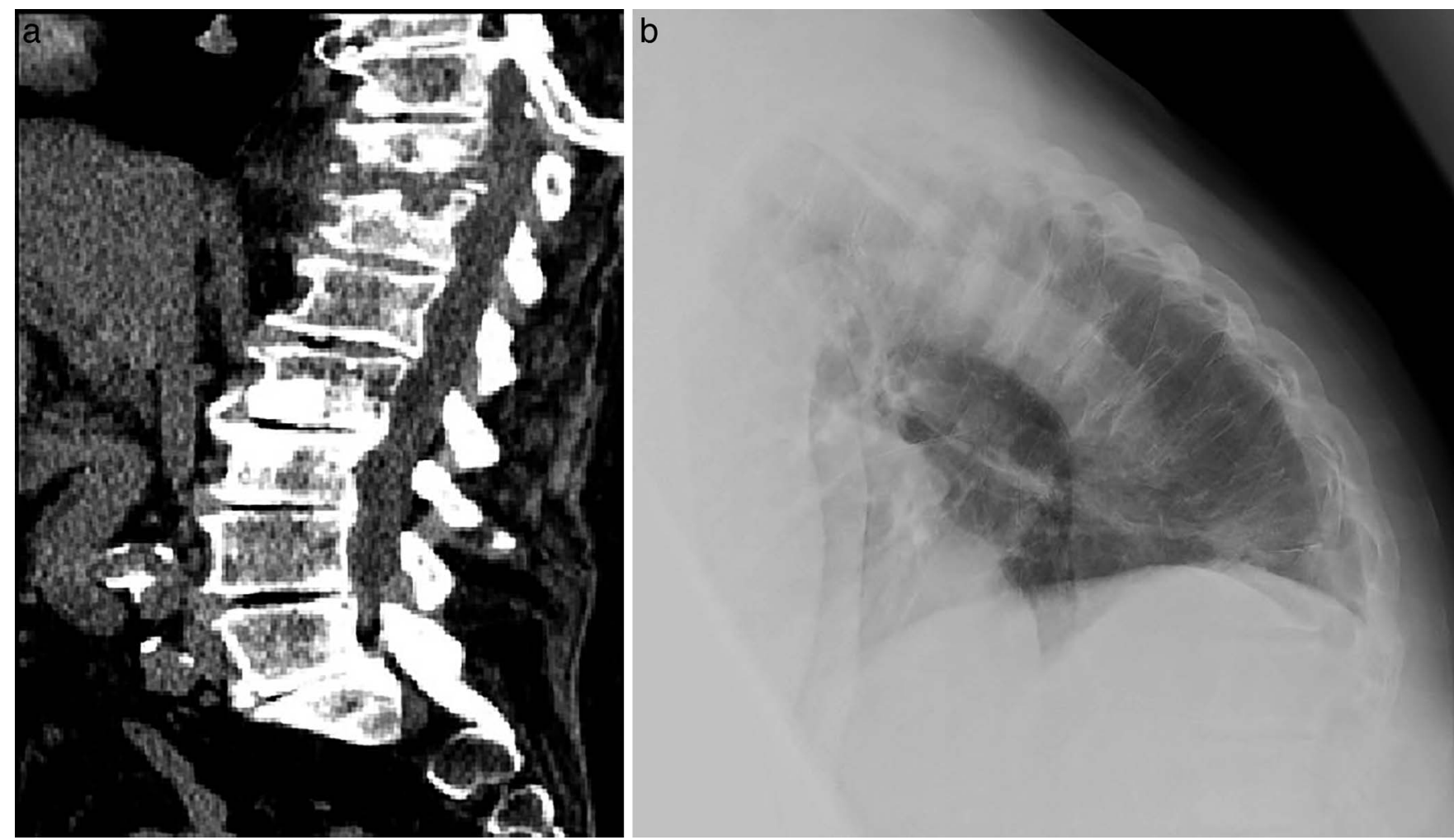

Figure 3. Kyphotic deformity. A 78-year-old healthy woman with 2 weeks of lower back pain. Her computed tomography revealed destruction of the T11 to T12 disc space and adjacent endplates (a) and, upon standing lateral radiograph, a kyphotic deformity of $75^{\circ}$ (b) accompanied by bilateral lower extremity weakness.

resolve under IV antibiotic treatment, but it continued with a fulminant course. These spondylodiscitis patients differ from traditional discitis patients in that despite being generally healthy patients with spontaneous spondylodiscitis, all but 1 patient were refractory to IV antibiotic treatment, deteriorated, and took on a fulminant clinical course.

While in the ward with bed rest and high-dose culture-specific IV antibiotic treatment, almost all patients continued to deteriorate neurologically and/or biomechanically, and catastrophic complications were often encountered, with obvious failure of conventional antibiotic treatment. An extension of the intradiscal infectious phlegmon through the posterior disc annulus and into the spinal canal occurred in 14 patients (Figure 4b). Patients with thoracolumbar and cervical infections had dominant neurologic symptoms, but patients with lumbar infections had a tendency to develop biomechanical instability, although they also experienced neurologic symptoms, including 1 case of acute cauda equina syndrome.

Patients required extensive and often repetitive surgical debridement because of neurologic and clinical deterioration, and expansion of the persistent infection with a mass effect. Patients required prolonged hospitalizations, ICU care, and longterm IV antibiotic treatment followed by extensive rehabilitation. The overwhelming majority of patients who ultimately required surgical intervention are in contrast to prior reports of spondylodiscitis in which the majority of patients were treated only with antibiotics and immobilization. ${ }^{4,8,9}$ Bettini et $\mathrm{al}^{3}$ reported excellent results with medical treatment alone, with only $3 \%$ of patients deteriorating and developing neurologic deficits and none reported to have developed epidural abscesses. Although the vast majority of reports support medical treatment of spondylodiscitis, a small number of studies have shown superior results with surgical treatment. ${ }^{10,11}$ In this study, despite multiple medical and surgical interventions, catastrophic complications were encountered and 3 patients died within 6 months, which is unequaled in prior reports on spondylodiscitis, but on the other hand may be comparable with prior reports of SEA. ${ }^{12}$

One possible explanation for this atypical clinical course may be related to the frequent extension of this entity from an initial formal 

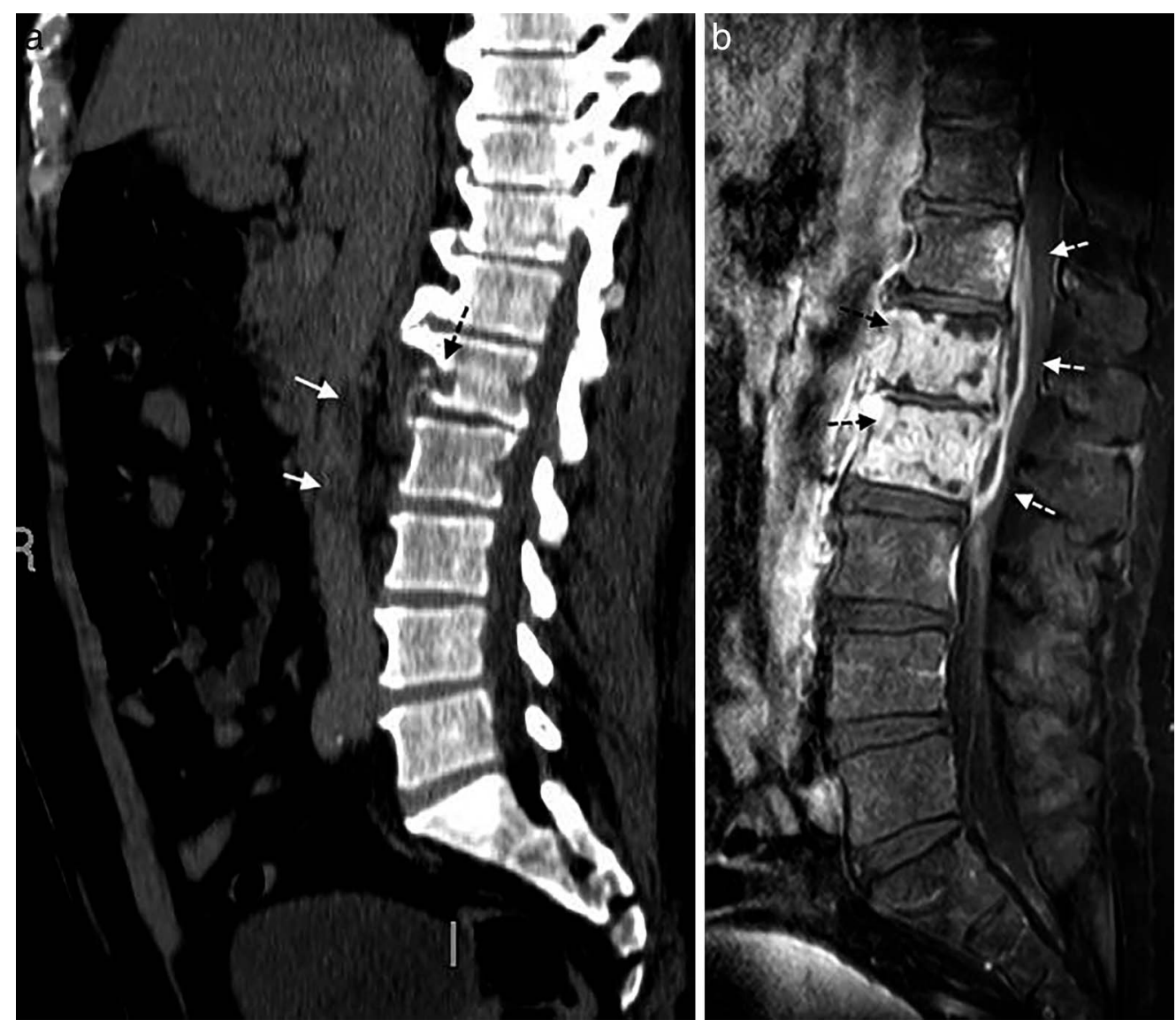

Figure 4. Fulminant spondylodiscitis. A healthy 55-year-old man with spondylodiscitis of L1 to L2 (black dashed arrow in a and b) that was cultured via computed tomography-guided biopsy. Note the inferior vena cava septic thrombi (white arrows in a). While under culture-specific IV antibiotic treatment, an extension through the posterior annulus and posterior longitudinal ligament to the epidural space occurred, essentially evolving into an unusual type of spinal epidural abscess, which is anterior to the dura, as shown on magnetic resonance imaging (white dashed arrows in b).

discitis, that at presentation was confined within the disc's anatomic borders, to an intracanal infection via direct posterior extension through the posterior annulus and PLL to the epidural

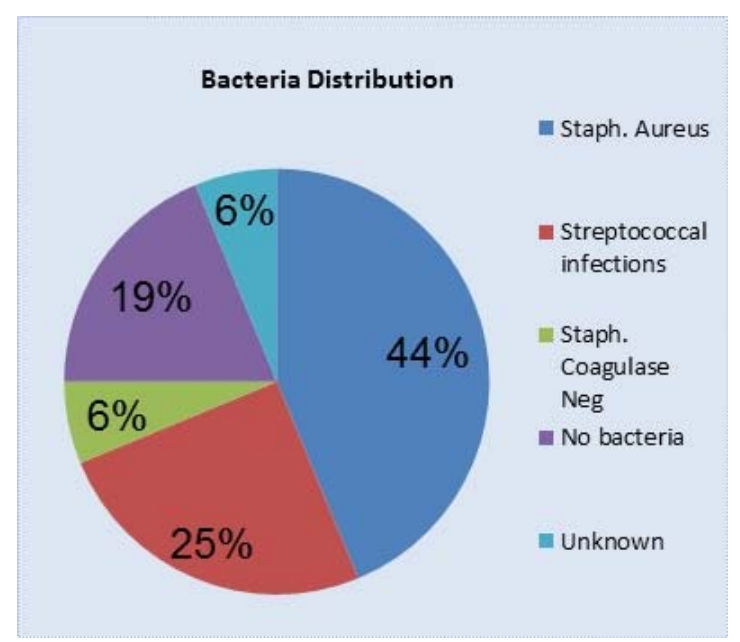

Figure 5. Bacteria distribution. When considering only positive cultures, Staphylococcus aureus represents approximately $60 \%$ of cases, which is comparable to previous reports in large series. space, essentially evolving into an unusual type of SEA that is anterior to the dura. This occurred in 14 of the 15 spondylodiscitis patients (93\%) during hospitalization while being treated with IV culture-

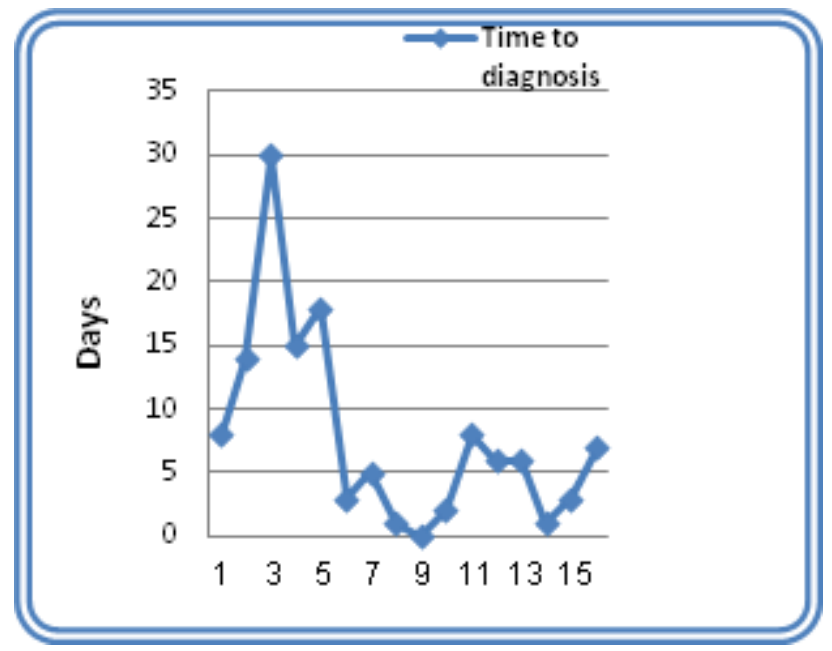

Figure 6. Time to diagnosis. A learning curve showing a reduction in time to diagnosis of spinal infections during the course of the study period, presumably due to more vigilant attention to these entities. 
specific antibiotics. Classic spondylodiscitis is usually defined as an infection confined to the intervertebral disc space and adjacent vertebrae, whereas this study exemplifies a more fulminant disease in which the posterior annulus of the disc and the PLL are breached and an anterior epidural abscess is formed (Figure 4b). This is unusual in SEA because most epidural abscesses are formed posterior to the dura adjacent to the ligamentum flavum and with no relation to the anterior intervertebral disc. This anterior SEA is presumably due to ongoing local deterioration with extension of the intradiscal infectious phlegmon through the posterior disc annulus and into the spinal canal that ultimately led to neurologic compromise. This finding was confirmed on MRI and during surgery. Extension of the infectious mass from disc infection to the spinal canal is reportedly extremely rare; most studies report no such occurrences, ${ }^{3,13,14}$ whereas some do mention atypical cases of this extension ranging between $10 \%$ and $16 \%$ of the patients. ${ }^{2,4,12}$ In contrast to 15 patients $(94 \%)$ who presented with the above entity, only 1 patient presented with classic SEA, in which the abscess formed in the posterior spinal column. ${ }^{15}$

Finally, another point of interest encountered in this study was the learning curve at our institution regarding recognition and diagnosis of deep spinal infections (Figure 6). Average time to diagnosis was 14.5 days (range, 3-30 days) during the first 2 years of the study, compared with a shorter time to diagnosis (average, 4 days; range, 0-8 days) in the last year of study. Time to diagnosis has been shown to be an independent prognostic factor, with longer delays leading to adverse outcomes. ${ }^{12}$ In this study such an association was not found, although it can be attributed to the small sample size. The limitations of this study are evident and related to its retrospective nature and the relatively small number of patients. Initial IV antibiotic treatment did follow a specific infectious disease protocol, yet the surgical treatment was patient tailored depending on disease severity, and this did not follow any particular protocol. Furthermore, it has not escaped our attention that this consecutive series of patients demonstrated an extremely irregular clinical course for which we currently lack an epidemiologic or bacteriologic explanation.

Despite the small number of patients in this series, the importance of this study lies in demon- strating the fulminant deterioration that may occur in antibiotic-resistant spondylodiscitis. Increased vigilance and early recognition of this condition and its misleading initial presentations are warranted.

\section{REFERENCES}

1. Zimmerli W. Clinical practice: vertebral osteomyelitis. $N$ Engl J Med. 2010;362(11):1022-1029.

2. Butler JS, Shelly MJ, Timlin M, Powderly WG, O'Byrne JM. Nontuberculous pyogenic spinal infection in adults: a 12year experience from a tertiary referral center. Spine (Phila Pa 1976). 2006;31(23):2695-2700.

3. Bettini N, Girardo M, Dema E, Cervellati S. Evaluation of conservative treatment of nonspecific spondylodiscitis. Eur Spine J. 2009; 18(suppl 1):143-150.

4. O'Daly BJ, Morris SF, O'Rourke SK. Long-term functional outcome in pyogenic spinal infection. Spine (Phila $P a$ 1976). 2008;33(8):E246-E253.

5. Bluman EM, Palumbo MA, Lucas PR. Spinal epidural abscess in adults. J Am Acad Orthop Surg. 2004;12(3):155-163.

6. Tang HJ, Lin HJ, Liu YC, Li CM. Spinal epidural abscess-experience with 46 patients and evaluation of prognostic factors. J Infect. 2002;45(2):76-81.

7. Davis DP, Salazar A, Chan TC, Vilke GM. Prospective evaluation of a clinical decision guideline to diagnose spinal epidural abscess in patients who present to the emergency department with spine pain. J Neurosurg Spine. 2011;14(6):765770 .

8. Carragee EJ. Pyogenic vertebral osteomyelitis. J Bone Joint Surg Am. 1997;79(6):874-880.

9. Curry WT, Hoh BL, Amin-Hanjani S, Eskandar EN. Spinal epidural abscess: clinical presentation, management, and outcome. Surg Neurol. 2005;63(4):364-371; discussion 371.

10. Woertgen C, Rothoerl RD, Englert C, Neumann C. Pyogenic spinal infections and outcome according to the 36item short form health survey. $J$ Neurosurg Spine. 2006;4(6):441-446.

11. Heyde CE, Boehm H, El Saghir H, Tschöke SK, Kayser R. Surgical treatment of spondylodiscitis in the cervical spine: a minimum 2-year follow-up. Eur Spine J. 2006;15(9):1380-1387.

12. McHenry MC, Easley KA, Locker GA. Vertebral osteomyelitis: long-term outcome for 253 patients from 7 Cleveland-area hospitals. Clin Infect Dis. 2002;34(10):13421350.

13. Karadimas EJ, Bunger C, Lindblad BE, Hansen ES, Høy K, Helmig P, et al. Spondylodiscitis: a retrospective study of 163 patients. Acta Orthop. 2008;79(5):650-659.

14. Schinkel C, Gottwald M, Andress HJ. Surgical treatment of spondylodiscitis. Surg Infect (Larchmt). 2003;4(4):387-391.

15. Rosinsky PJ, Zimhony O, Ciobotaro P, Sagiv S, BenGalim P. Intradural extension of a pyogenic epidural abscess. JBJS Case Connect. 2015;5(3):e76.

Disclosures and COI: All authors certify that they have nothing to disclose in relation to this study and certify that none of the authors have any financial and/or personal relationships with other 
people or organizations that could inappropriately influence this work (employment, consultancies, stock ownership, honoraria, paid expert testimony, patent applications/registrations, and grants or other funding).

Corresponding Author: Philip Rosinsky, MD, Department of Orthopaedic Surgery, Kaplan Medical Center, Pasternak St, POB 1, Rehovot
76100, Israel. Phone: +972-8-9441340; Fax: +972-89411605; E-mail: prosinsky@gmail.com.

Published 21 December 2018

This manuscript is generously published free of charge by ISASS, the International Society for the Advancement of Spine Surgery. Copyright (C) 2018 ISASS. To see more or order reprints or permissions, see http://ijssurgery.com. 